\title{
Fear Avoidance Beliefs and Kinesiophobia Are Presented in Ath- letes who Suffer from Gastrocnemius Chronic Myofascial Pain [AQ1]
}

San-Antolín et al.

Athletes' Myofascial Pain Kinesiophobia \& Fear Avoidance

\section{Marta San-Antolín MSc, PSYCH, ${ }^{*}$ David Rodríguez-Sanz PhD, MSc, PT, DP, ${ }^{\dagger}$ Davinia Vicente- Campos PhD, MSc, PT ${ }^{\ddagger}$ Patricia Palomo-López PhD, MSc, DP $§$ Carlos Romero-Morales PhD, MSc, PT ๆ María Benito-de-Pedro PhD, MSc, PT, DP, ${ }^{\dagger}$ Daniel López-López PhD, MSc, DP, II and César Calvo-Lobo PhD, MSc, PT"l [AQ2]}

*. Department of Psychology, Universidad Europea de Madrid, Villaviciosa, de Odón, Madrid, Spain

${ }^{+}$. Facultad de Enfermería, Fisioterapia y Podología, Universidad Complutense de Madrid, Madrid, Spain

¥. Facultad de Ciencias de la Salud, Universidad Francisco de Vitoria, Pozuelo de Alarcón, Madrid, Spain

$\S$. University Center of Plasencia, Universidad de Extremadura, Badajoz, Spain

9. Faculty of Sport Sciences, Universidad Europea de Madrid, Villaviciosa, de Odón, Madrid, Spain

II. Research, Health and Podiatry Unit, Department of Health Sciences, Faculty of Nursing and Podiatry, Universidade da Coruña, Spain

Funding sources: There was no funding.

Conflicts of interest: There are no conflicts of interest.

Correspondence to: David Rodríguez-Sanz, PhD, MSc, PT, DP, Facultad de Enfermería, Fisioterapia y Podología, Universidad Complutense de Madrid, Madrid, Spain. E-mail: davidrodriguezsanz@ucm.es. [AQ3]

\section{ABSTRACT}

To compare and predict kinesiophobia and fear avoidance beliefs between athletes with gastrocnemius myofascial pain syndrome (MPS) and healthy athletes.

Case-control.

Outpatient clinic.]

Fifty athletes were $\equiv \mathrm{d}$ into athletes with chronic gastrocnemius MPS $(\mathrm{N}=25)$ and healthy athletes $(\mathrm{N}=25)$.

Kinesiophobia symptoms total and domain scores (harm and activity avoidance) and levels were determined by the Tampa Scale of Kinesiophobia (TSK-11). Fear avoidance beliefs total and domain scores (physical and working activities) were measured by the Fear Avoidance Beliefs Questionnaire (FABQ).

Significant differences $(P<0.05)$ with a large effect size $(d=0.81-4.22)$ were found between both groups, with greater kinesiophobia symptom scores for the TSK-11 activity avoidance domain and total scores, and greater fear avoidance beliefs scores for the FABQ physical and working activities domains and total scores of athletes with gastrocnemius MPS with respect to healthy athletes. TSK-11 total score showed[AQ4] a prediction model $\left(R^{2}=0.256\right)$ based on the FABQ total score. The FABQ total score showed a prediction model $\left(R^{2}=0.741\right)$ based on gastrocnemius MPS presence $\left(R^{2}=0.665\right)$, levels of kinesiophobia $\left(R^{2}=0.052\right)$, and height $\left(R^{2}=0.025\right)$.

Greater kinesiophobia levels, greater total and activity avoidance domain scores (but not for the harm domain), and greater fear avoidance beliefs total and domain scores (work and physical activity) were shown for athletes with gastrocnemius MPS vs healthy athletes. Higher kinesiophobia symptoms were predicted by greater fear avoidance beliefs in athletes. Greater fear avoidance beliefs were predicted by the presence of gastrocnemius MPS, higher levels of kinesiophobia, and lower height in athletes.

Keywords: Athletes ; Chronic Pain ；Musculoskeletal Pain ; Myofascial Pain Syndrome ; Sports ; Trigger Points 


\section{Introduction}

Myofascial pain syndrome (MPS) may be defined as and considered a common musculoskeletal condition that comprises signs and symptoms due to the presence of myofascial trigger points (MTrPs). These points present hyperirritable nodules in skeletal muscular taut bands, producing typical local and/or referred pain in conjunction with other autonomic, motor[AQ5], and/or sensitive alterations [1,2].

Psychological conditions such as kinesiophobia and pain-related fear seem to be commonly presented in athletes suffering from pain alterations, playing a key role in the intensity and frequency of soreness as well as having physical and/or psychological consequences in athletic populations [3-5]. Indeed, athletes who suffer from patellofemoral pain present higher kinesiophobia with respect to healthy controls [6]. In addition, athletes with a history of anterior cruciate ligament reconstruction who have not participated in sports present greater fear avoidance beliefs with respect to athletes with a history of anterior cruciate ligament reconstruction who have participated in sports or healthy athletes. Also, athletes with a history of anterior cruciate ligament reconstruction who have participated in sports present greater fear avoidance related to work and physical activities compared with healthy athletes [5]. According to these findings, additional psychosocial care should be specially directed toward controlling fear avoidance beliefs and kinesiophobia in athletes suffering from pain disorders and returning to sports [5,6]. Despite gastrocnemius MTrPs having recently been a main focus of intervention in athletes [7, 8], there is a lack of research evidence regarding fear avoidance beliefs and kinesiophobia in athletes suffering from gastrocnemius MPS.

Indeed, MTrPs may be considered peripheral pain generators for general and regional musculoskeletal pain conditions $[9,10]$ associated with local ischemia, low $\mathrm{pH}$, and release of inflammatory mediators [11]. Regarding MTrPs' clinical diagnosis, MTrPs may be divided into latent or active. Both types seem to present the same characteristics, although only active MTrPs are associated with spontaneous pain and patients' pain recognition during stimulation $[1,2,9,10]$. MTrPs show a high prevalence (from $13 \%$ to $30 \%$ ) in gastrocnemius muscles under triceps surae musculoskeletal alterations $[12,13]$. Furthermore, lower limb musculoskeletal conditions are the reason for up to one-third of medical sport consultations [14]. Concretely, gastrocnemius musculoskeletal alterations are the most common injuries in the lower limb muscles of athletes during training (up to 39\%) and competition (up to 46\%) [15]. [AQ6]

According to prior studies, the role of kinesiophobia and fear avoidance beliefs could be important in MPS interventions [16-18]. A high prevalence of gastrocnemius muscle injuries have been previously shown in athletes [14, 15, 19], including frequently active MTrP-generating MPS [12, 13]. Consequently, MPS interventions have been recently applied to MTrPs of gastrocnemius in athletes [7, 8]. Fear avoidance beliefs and kinesiophobia may play a key role in athletes suffering from pain conditions $[5,6]$. According to these considerations, the influence of fear avoidance beliefs and kinesiophobia needs to be deeply studied in athletes suffering from gastrocnemius MPS. Thus, the main purpose of this research was to compare kinesiophobia symptoms and levels between athletes suffering from gastrocnemius MPS and healthy athletes. Furthermore, another secondary aim was to compare fear avoidance belief scores between athletes suffering from gastrocnemius MPS and healthy athletes. Finally, other secondary aims were to predict kinesiophobia symptoms according to descriptive data, fear avoidance scores, and MPS presence, as well as predicting fear avoidance beliefs based on descriptive data, kinesiophobia scores and levels, and MPS presence. Therefore, it was hypothesized that athletes suffering from gastrocnemius MPS could exhibit higher kinesiophobia symptoms and levels and fear avoidance belief scores compared with healthy athletes. Furthermore, it was hypothesized that kinesiophobia symptom scores could be predicted based on descriptive data, fear avoidance scores, and MPS presence, and that fear avoidance belief scores could be predicted based on descriptive data, scores and levels of kinesiophobia, and MPS presence.

\section{Methods}

\section{Design}

The study design was carried out as a case-control secondary analysis according to the STrengthening the Reporting of OBservational studies in Epidemiology (STROBE) guidelines [20, 21]. Indeed, kinesiophobia symptoms and fear avoidance beliefs were analyzed comparing athletes suffering from MPS vs healthy athletes using self-reported questionnaires. The Ethical Committee of The Princess University Hospital (CEIm 22/18) approved this study. Be- 
fore starting this study, athletes signed the informed consent form. Finally, the Helsinki Declaration and human experimentation ethical requirements were respected during the study's course [22].

\section{Calculation of Sample Size}

Kinesiophobia score was considered the main outcome measurement in order to perform the sample size calculation, as athletes suffering from musculoskeletal pain conditions in the lower limbs seemed to present greater kinesiophobia symptoms with respect to healthy controls [6]. G*Power software (version 3.1.9.2; Dusseldorf University, Dusseldorf, Germany) was utilized to perform the sample size calculation using the between two independent groups difference [23]. Kinesiophobia total score, assessed with the Spanish validated Tampa Scale of Kinesiophobia-11 items (TSK-11) $[24,25]$ from a pilot study $(\mathrm{N}=17)$ with two groups described using mean $\pm \mathrm{SD}$, including seven athletes suffering from gastrocnemius MPS $(22.71 \pm 4.92$ points $)$ and seven healthy athletes (19.14 \pm 4.71 points), was utilized to perform the sample size calculation. In addition, an effect size of $d=0.74$, error probability of $\alpha=0.05,95 \%$ confidence interval, power of $1-\beta$ error probability $=0.80$ ), one-tailed hypothesis, and allocation ratio of $\mathrm{N} 2 / \mathrm{N} 1=1$ were used to calculate the sample size. Thus, a total sample size of 48 athletes, 24 athletes for each group, was determined. According to a possible 5\% loss to follow-up, a total sample size of 50 athletes, divided into 25 athletes suffering from MPS and 25 healthy athletes, was used.

\section{Sample}

A total sample of 50 athletes was matched pairwise for descriptive data and recruited by a consecutive convenience sampling method from 2018 to 2019. Athletes were divided into cases, including patients suffering from chronic gastrocnemius MPS for more than three months $(\mathrm{N}=25$; gastrocnemius MPS group), and controls, including healthy matched paired participants $(\mathrm{N}=25$; healthy group).

Athletes who carried out level II (moderate) or III (vigorous) physical activity according to scores $>600$ $\mathrm{MET} / \mathrm{min} / \mathrm{wk}$, measured by the International Physical Activity Questionnaire (IPAQ), with an age range from 18 to 65 years were included in the study [26]. First, the control group included healthy athletes matched pairwise for descriptive data (sociodemographic, physical activity, and sport category characteristics) without MPS or other conditions at least for the previous year [27, 28]. Second, the case group was comprised of athletes suffering from bilateral or unilateral gastrocnemius MPS suffering from persisting and chronic pain for more than three months and presenting at least one active MTrP in the gastrocnemius diagnosed by an experienced physical therapist according to a standardized manual palpation procedure with good agreement reliability $(\kappa=0.63)$ [29]. The presence of three positive signs/symptoms from four possible items, including 1) sensible knot, 2) taut band, 3) pain pattern recognition during stimulation by palpation and presence of spontaneous pain (mandatory criterion for an active MTrP), and 4) ankle dorsiflexion limitation at full stretch, were used for active MTrP diagnosis [1, 2, 30].

Systemic conditions, neurological disorders, arthritis, neoplasms, autoimmune alterations, vascular pathologies, neuropathic conditions (e.g., radiculopathy), fractures, tendinopathies, sprains, surgeries, lower limb dysmetria (difference $>1 \mathrm{~cm}$ between both lower limbs), mental disorders, and cognitive alterations were assessed by the questionnaires during the study's course $[12,13,31]$.

\section{Descriptive Data}

Body mass index (BMI was calculated by the Quetelet index as weight $/$ height ${ }^{2}$ and measured as $\mathrm{kg} / \mathrm{cm}^{2}$ ) [32], height (measured by $\mathrm{cm}$ ), weight (measured by $\mathrm{kg}$ ), sex (categorized as male or female), age (measured by years), main sport category performance (categorized as running, fitness, soccer, athletics, or paddle), pain intensity (measured by the visual analog scale) [33], and pain location (categorized as left, right, or bilaterally gastrocnemius) were collected [27].

In addition, the International Physical Activity Questionnaire (IPAQ) was self-reported by all study participants [26]. This questionnaire was used to report physical activity scores and levels by the index of metabolic equivalents per minutes per week $(\mathrm{MET} / \mathrm{min} / \mathrm{wk})$; scores were categorized into moderate physical activity $(600-1,500$ $\mathrm{MET} / \mathrm{min} / \mathrm{wk})$ or vigorous physical activity $(\geq 1,500 \mathrm{MET} / \mathrm{min} / \mathrm{wk})$ level, respectively. This tool showed good psychometric properties for repeatability (Spearman's $r h o=0.80$ ) and criterion validity (median $r h o=0.30$ ) [34]. 


\section{Outcome Measurements}

The main outcome measurement was the kinesiophobia total score, assessed with the Spanish validated Tampa Scale of Kinesiophobia-11 items [24, 25]. Secondary outcome measurements were harm and activity avoidance kinesiophobia domain scores as well as kinesiophobia levels assessed with the TSK-11 [24, 25], and the total scores as well as working and physical activity domain scores of the fear avoidance beliefs symptoms measured with the Spanish validated Fear Avoidance Beliefs Questionnaire (FABQ) [35, 36]. Both tools were self-reported by case and control athletes with the support of a psychologist with clinical experience of 10 years in order to clarify doubts when filling out these questionnaires.

\section{Tampa Scale of Kinesiophobia-11 Items}

The Spanish validated TSK-11 was self-reported by case and control athletes to determine the kinesiophobia symptoms total score, harm and activity avoidance kinesiophobia domain scores, and categorization into levels of fear of movement $[24,25]$. Kinesiophobia may be defined as an initial adaptive response to threat that may produce maladaptive and avoidance behaviors, increasing fear and pain and producing limited activity and fear of movement [37-39]. Fear of movement may be a predictor of future disability [37]. This scale presented a total kinesiophobia symptoms score and two domains: activity avoidance and harm. This tool was scored by a four-point Likert scale, with higher scores representing greater fear of pain, movement, and injury. TSK-11 total scores were divided into levels of fear of movement: no fear (from 0 to 17 points), slight fear (from 18 to 24 points), moderate fear (from 25 to 31 points), severe fear (from 32 to 38 points), and maximum fear (from 39 to 44 points) [24, 25]. Acceptable psychometric properties have been shown with good internal consistency (Cronbach's $\alpha=0.78$ ), test-retest (intraclass correlation coefficient $[\mathrm{ICC}]=0.82)$, standard error of measurement $(\mathrm{SEM}=3.16)$, responsiveness $(-1.19)$, minimum clinically important difference (MCID = 4.80), and minimum detectable change (MDC = 5.60) $[24,25,40-42]$.

\section{Fear Avoidance Beliefs Questionnaire}

The FABQ was used in the present study to evaluate the influence of fear avoidance beliefs on daily disability secondary to pain presence in athletes who carry out physical activity while suffering from a chronic MPS condition $[43,44]$. This tool provided a total score of fear avoidance beliefs symptoms and was divided into two domains, fear avoidance beliefs related to physical activity (FABQ-PA) and working activity (FABQ-W), including a total of 16 items in a Likert-type scale from 0 to 6 points, with $0=$ "totally disagree" and $6=$ "totally disagree." The total range varied from 0 to 55 points. Higher score values showed greater fear of pain and physical activity, resulting in more avoidance behaviors. The Spanish validated FABQ showed acceptable psychometric properties, with a Cronbach $\alpha$ internal consistency of 0.91, an ICC of 0.96, and a Kappa value mean for all items of 0.74 [45].

\section{Statistical Analysis}

The Statistical Package for Social Sciences (version 24.0; IBM Corp., Armonk, NY, USA) was used to analyze all data, with an $\alpha$ error of 0.05 and a $P$ value $<0.05$ considered statistically significant, in line with the detailed sample size calculation.

For quantitative data analyses, Shapiro-Wilk tests were used to calculate normality distributions. Data were analyzed as parametric if they were adjusted to normal distributions showing a $P$ value $\geq 0.05$ on the Shapiro-Wilk test. Data were analyzed as nonparametric if they were not adjusted to normal distributions showing a $P$ value $<0.05$ on the Shapiro-Wilk test. Parametric data were described as mean \pm SD and range (minimum to maximum values), and the differences between athletes with gastrocnemius MPS and healthy athletes were analyzed by the Student $t$ test for independent samples according to Levene's test for equality of variances. Nonparametric data were described as median \pm SD and range (minimum to maximum values), and the differences between athletes with gastrocnemius MPS and healthy athletes were analyzed by the Mann-Whitney $U$ test for independent samples. For all outcome measurements, effect size was calculated by Cohen's $d$ and divided into very small $(d<0.20)$, small $(d=0.20-0.49)$, medium $(d=0.50-0.79)$, and large effect sizes $(d>0.8)[46]$.

Considering categorical data analyses, data were described as frequency (No.) and percentage (\%), and the differences between athletes with gastrocnemius MPS and healthy athletes were analyzed according to the Fisher exact test for dichotomous data or the chi-square test for polytomous data. 
Afterward, multivariate predictive analyses were carried out by two linear regression models. Both linear regression models were performed by applying stepwise selection methods and $R^{2}$ coefficients to establish the quality of adjustment [47]. The first linear regression model used descriptive data such as sex, age, height, weight, BMI, IPAQ scores and categories, main sport category performance, and gastrocnemius MPS presence (case or control group, fear avoidance beliefs measured by the FABQ domains (related to physical and working activities) and total score as independent variables. Kinesiophobia symptoms measured by TSK-11 total scores were included as the dependent variable. The second linear regression model also used descriptive data such as sex, age, height, weight, BMI, IPAQ scores and categories, main sport category performance, gastrocnemius MPS presence (case or control group), and kinesiophobia symptoms and levels measured by the TSK-11 domains (related to harm and activity avoidance) and total score and their categorization into levels of fear (no, slight, moderate, severe, and maximum fear levels) as independent variables. Fear avoidance beliefs measured by the FABQ total score were included as the dependent variable. Pre-established parameters of the F probability were $P_{\text {in }}=0.05$ and $P_{\text {out }}=0.10$, and $P$ values $<0.05$ were considered statistically significant according to all statistical analyses.

\section{Results}

\section{Descriptive Data}

From a total sample of 72 athletes evaluated for eligibility, 22 athletes were excluded due to presence of systemic disorders such as diabetes $(\mathrm{N}=3)$, radiculopathy $(\mathrm{N}=1)$, vascular alterations $(\mathrm{N}=3)$, rheumatoid arthritis $(\mathrm{N}=4)$, prior lower limb fractures and/or surgeries $(\mathrm{N}=4)$, Achilles and/or patellar tendinopathy $(\mathrm{N}=3)$, level I physical activity according to the IPAQ $(\mathrm{N}=2)$, and absence of at least one active $\mathrm{MTrP}$ in the gastrocnemius muscle $(\mathrm{N}=2)$ according to the flow diagram (Figure 1). A total sample of 50 athletes was included in this study and divided into athletes with chronic gastrocnemius MPS ( $\mathrm{N}=25$; MPS case group) and healthy athletes $(\mathrm{N}=25$; healthy control group). Descriptive data did not show statistically significant differences $(P>0.05)$ between athletes with gastrocnemius MPS vs healthy athletes for sex (13 women and 12 men vs 10 women and 15 men, $P=0.571)$, age ( $38.00 \pm 11.84$ vs $41.00 \pm 11.72$ years, $P=0.509)$, weight $(69.76 \pm 10.70$ vs $68.28 \pm 14.48 \mathrm{~kg}, P=0.683)$, height $(1.71 \pm 0.10$ vs $1.69 \pm 0.10 \mathrm{~m}, P=0.580)$, BMI $\left(23.64 \pm 2.44\right.$ vs $\left.23.40 \pm 2.87 \mathrm{~kg} / \mathrm{cm}^{2}, P=0.759\right)$, IPAQ score $(2,946.00 \pm 2,938.50$ vs $2,277.00 \pm 3,746.50 \mathrm{MET} / \mathrm{min} / \mathrm{wk})$, IPAQ category (seven moderate and 18 vigorous vs 11 moderate and 14 vigorous physical activity level), and main sport category performance (six running, 10 fitness, four soccer, two athletics, and three paddle vs nine running, 13 fitness, two soccer, one athletics, and zero paddle sport), respectively. In addition, the group of athletes suffering from MPS showed a pain intensity of $5.70 \pm 3.05$ points, located at the right gastrocnemius in 10 athletes, left gastrocnemius in three athletes, and bilaterally in 12 athletes.

Figure 1 Flow diagram.[AQ14]

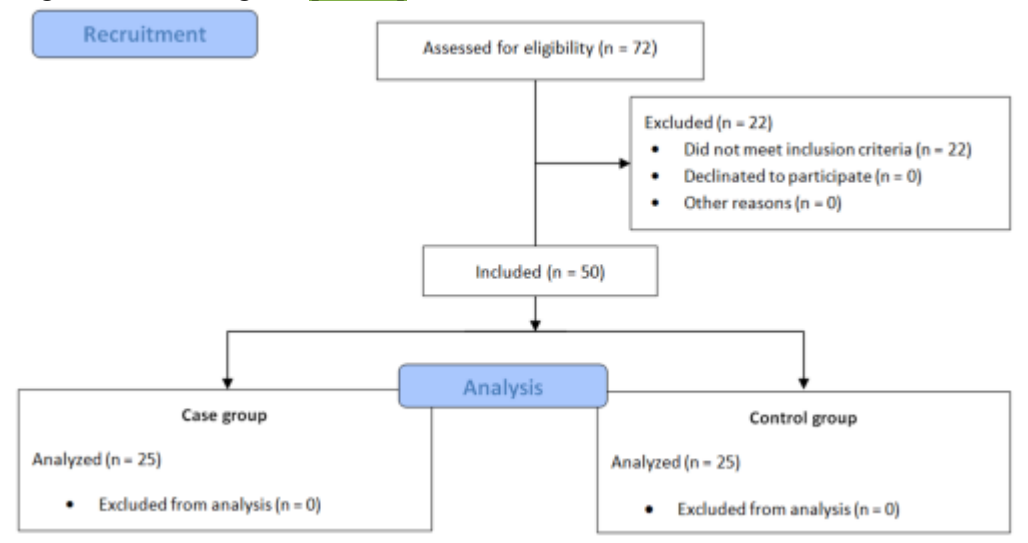

Figure 2 Distribution graph showing TSK-11 scores and kinesiophobia cutoff scores showing levels of fear such as no fear (0-17 points), slight fear (18-24 points), moderate fear (25-31 points), severe fear (32-38 points), and maximum fear (39-44 points) levels for athletes suffering from gastrocnemius MPS and healthy athletes. MPS = myofascial pain syndrome; TSK-11 = Tampa Scale of Kinesiophobia-11 items. [AQ13] 


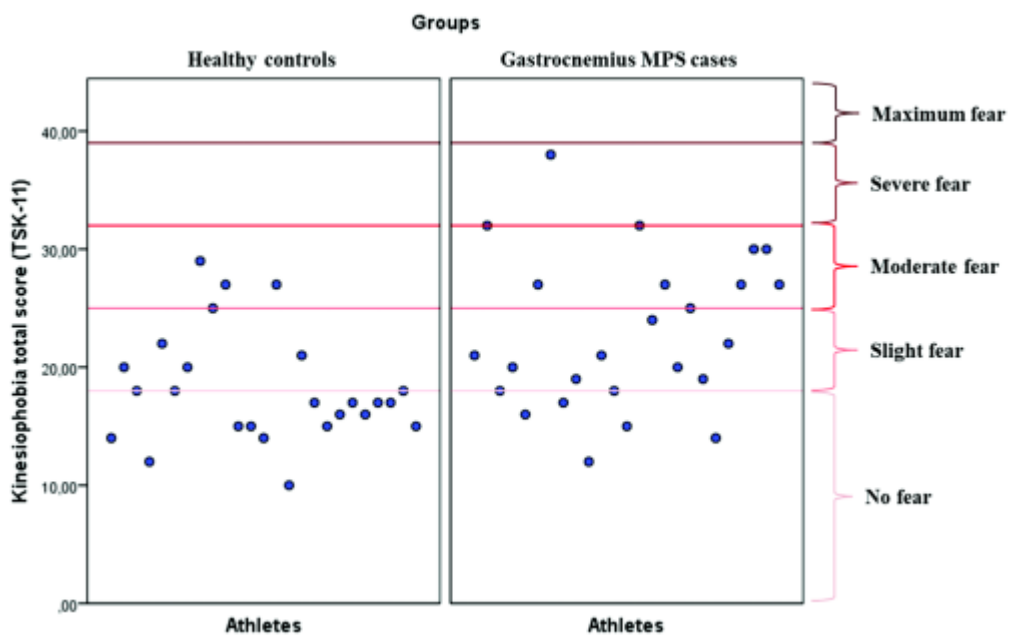

\section{Kinesiophobia}

Statistically significant differences $(P<0.01)$ with a large effect size $(d=0.81-0.87)$ were shown between the groups, detailing greater kinesiophobia symptom scores for the TSK-11 activity avoidance domain and total scores of athletes suffering from gastrocnemius MPS with respect to healthy athletes, although there were no statistically significant differences $(P=0.145)$, with a small effect size $(d=0.41)$ for the harm domain scores of the kinesiophobia symptom scores. In addition, statistically significant differences $(P=0.035)$ were shown for TSK-11 categorization presenting higher levels of fear of movement for the athletes with gastrocnemius MPS with respect to healthy athletes (Table 1; Figure 1).

Table 1 Kinesiophobia symptom scores and levels according to the domains, total score, and categorization of TSK-11 for athletes suffering from gastrocnemius MPS, healthy athletes, and the total sample

\begin{tabular}{|c|c|c|c|c|c|}
\hline Kinesiophobia (TSK-11) & Total Group & $\begin{array}{l}\text { Gastrocnemius MPS } \\
\text { Cases } \\
(\mathrm{N}=25)\end{array}$ & $\begin{array}{l}\text { Healthy Control } \\
(\mathrm{N}=25)\end{array}$ & $\begin{array}{l}P \text { MPS vs } \\
\text { Healthy }\end{array}$ & Effect Size, $d$ \\
\hline \multirow[t]{2}{*}{ Activity avoidance } & $12.00 \pm 5.00$ & $14.00 \pm 7.50$ & $11.00 \pm 3.50$ & \multirow[t]{2}{*}{$0.008 *$} & \multirow[t]{2}{*}{0.87} \\
\hline & $(6.00-24.00)$ & $(8.00-24.00)$ & $(6.00-19.00)$ & & \\
\hline \multirow[t]{2}{*}{ Harm } & $7.00 \pm 4.00$ & $8.00 \pm 3.50$ & $7.00 \pm 5.00$ & \multirow[t]{2}{*}{$0.145^{*}$} & \multirow[t]{2}{*}{0.41} \\
\hline & $(4.00-16.00)$ & $(4.00-16.00)$ & $(4.00-14.00)$ & & \\
\hline \multirow[t]{2}{*}{ Total score } & $19.00 \pm 9.50$ & $21.00 \pm 9.00$ & $17.00 \pm 5.50$ & \multirow[t]{2}{*}{$0.007 *$} & \multirow[t]{2}{*}{0.81} \\
\hline & $(10.00-38.00)$ & $(12.00-38.00)$ & $(10.00-29.00)$ & & \\
\hline \multirow[t]{5}{*}{ Fear levels } & No $(0-17)$ & $19(38 \%)$ & $5(20 \%)$ & $14(56 \%)$ & \multirow[t]{5}{*}{$\mathbf{0 . 0 3 5}{ }^{\dagger} \mathrm{N} / \mathrm{A}$} \\
\hline & Slight (18-24) & $17(34 \%)$ & $10(40 \%)$ & $7(28 \%)$ & \\
\hline & Moderate (25-31) & $11(22 \%)$ & $7(28 \%)$ & $4(16 \%)$ & \\
\hline & Severe (32-38) & $3(6 \%)$ & $3(12 \%)$ & $0(0 \%)$ & \\
\hline & Maximum (39-44) & $0(0 \%)$ & $0(0 \%)$ & $0(0 \%)$ & \\
\hline
\end{tabular}

$P$ values $<0.05$ for a $95 \%$ confidence interval were considered statistically significant (bold).

MPS = myofascial pain syndrome; N/A = not applicable; TSK-11 = Tampa Scale of Kinesiophobia-11 items.

$*$ Median \pm interquartile range and range (minimum-maximum) and Mann-Whitney $U$ test were used. 
$†$ Frequency and percentage (\%) and chi-square test $\left(\chi^{2}\right)$ were used.

The TSK-11 total score showed a statistically significant prediction model $\left(R^{2}=0.256\right)$ based on the FABQ total score $\left(R^{2}=0.256, \beta=+0.163, \mathrm{~F}_{[1,48]}=16.557, P<0.001\right)$, predicting greater kinesiophobia symptom scores based on higher fear avoidance beliefs scores. Thus, the rest of the independent variables were excluded $(P>0.05)$ from the described prediction model due to TSK-11 total scores, as dependent variables were not predicted by sex, age, height, weight, BMI, IPAQ scores and categories, main sport category performance, gastrocnemius MPS presence (case or control group), or FABQ domains (related to physical and working activities) as independent variables following preestablished $\mathrm{F}$ probability parameters (Table 2 ).

Table 2 Linear regression model for the kinesiophobia total scores multivariate prediction between athletes suffering from gastrocnemius MPS and healthy athletes

\begin{tabular}{|c|c|c|c|}
\hline Parameter & Model & \multicolumn{2}{|c|}{$R^{2}$ Change Model $R^{2}$} \\
\hline TSK-11 total score & 17.509 & & \\
\hline & $+0.163 \times$ FABQ total score & $0.256^{*}$ & 0.256 \\
\hline
\end{tabular}

$\mathrm{FABQ}=$ Fear Avoidance Beliefs Questionnaire; MPS = myofascial pain syndrome; TSK-11 = Tampa Scale of Kinesiophobia - 11 items.

$* P$ values $<0.001$ for a $95 \%$ confidence interval are shown.

\section{Fear Avoidance Beliefs}

Statistically significant differences $(P<0.001)$ with a large effect size $(d=1.46-4.22)$ were shown between the groups, detailing greater fear avoidance beliefs scores for the FABQ physical and working activity domains and total scores of athletes suffering from gastrocnemius MPS with respect to healthy athletes (Table 3 ).

Table 3 Fear avoidance beliefs according to the FABQ domains and total scores of athletes suffering from gastrocnemius MPS, healthy athletes, and total sample

\begin{tabular}{|c|c|c|c|c|c|}
\hline \multirow[t]{2}{*}{$\begin{array}{l}\text { Fear Avoidance Beliefs } \\
\text { (FABQ) }\end{array}$} & Total Group & $\begin{array}{l}\text { Gastrocnemius MPS } \\
\text { Cases }\end{array}$ & Healthy Contr & \multirow[t]{2}{*}{ Healthy } & \multirow[t]{2}{*}{$\begin{array}{l}\text { Effect Size } \\
d\end{array}$} \\
\hline & $(\mathrm{N}=50)$ & $(\mathrm{N}=25)$ & $(\mathrm{N}=25)$ & & \\
\hline \multirow{2}{*}{ Physical activity } & $7.50 \pm 15.75$ & $18.00 \pm 5.00$ & $3.00 \pm 4.50$ & \multirow[t]{2}{*}{$<0.001 *$} & \multirow[t]{2}{*}{4.22} \\
\hline & $(0.00-25.00)$ & $(8.00-25.00)$ & $(0.00-7.00)$ & & \\
\hline \multirow[t]{2}{*}{ Working activity } & $2.00 \pm 17.25$ & $17.00 \pm 21.50$ & $0.00 \pm 2.00$ & \multirow[t]{2}{*}{$<0.001$ * } & \multirow[t]{2}{*}{1.46} \\
\hline & $(0.00-55.00)$ & $(0.00-55.00)$ & $(0.00-4.00)$ & & \\
\hline \multirow[t]{2}{*}{ Total score } & $10.00 \pm 31.75$ & $33.00 \pm 24.50$ & $3.00 \pm 5.00$ & \multirow[t]{2}{*}{$<0.001 *$} & \multirow[t]{2}{*}{2.45} \\
\hline & $(00.00-79.00)$ & $(8.00-79.00)$ & $(0.00-10.00)$ & & \\
\hline
\end{tabular}

$P$ values $<0.05$ for a $95 \%$ confidence interval were considered statistically significant (bold).

$\mathrm{FABQ}=$ Fear-Avoidance Beliefs Questionnaire $;$ MPS $=$ myofascial pain syndrome

*Median \pm interquartile range and range (minimum-maximum) and Mann-Whitney $U$ test were used.

The FABQ total score showed a statistically significant prediction model $\left(R^{2}=0.741\right)$ based on the gastrocnemius MPS presence according to groups $\left(R^{2}=0.665, \beta=+27.642, \mathrm{~F}_{[1,48]}=95.097, P<0.001\right)$, levels of fear of movement according to TSK-11 categorization $\left(R^{2}=0.052, \beta=+4.358, \mathrm{~F}_{[1,47]}=8.613, P=0.005\right)$, and height $\left(R^{2}=\right.$ $\left.0.025, \beta=-0.297, \mathrm{~F}_{[1,46]}=4.358, P=0.042\right)$, predicting greater fear avoidance belief scores for athletes suffering 
from gastrocnemius MPS, higher kinesiophobia levels, and lower height of athletes. Thus, the rest of the independent variables were excluded $(P>0.05)$ from the described prediction model due to FABQ total scores as dependent variables not being predicted by sex, age, weight, BMI, IPAQ scores and categories, or main sport category performance; nor were TSK-11 domains or total scores (related to harm and activity avoidance) used as independent variables due to the pre-established F probability parameters (Table 4). [AQ7]

Table 4 Linear regression model for the fear avoidance beliefs total scores multivariate prediction between athletes suffering from gastrocnemius MPS and healthy athletes

\begin{tabular}{|l|l|l|l|}
\hline Parameter & Model & \multicolumn{2}{|l|}{$R^{2}$ Change Model $R^{2}$} \\
\hline FABQ total score & 50.723 & & \\
\hline & $+27.642 \times$ MPS presence (group) & $0.665^{* *}$ & \\
\cline { 2 - 4 } & $+4.713 \times$ fear levels (TSK-11 categorization) & $0.052^{*}$ & \\
\cline { 2 - 4 } & $-0.297 \times$ height $(\mathrm{m})$ & $0.025^{*}$ & 0.741 \\
\hline
\end{tabular}

Multiplay: fear levels (TSK-11 categorization as: no fear $=0$, slight fear $=1$, moderate fear $=2$, severe fear $=3$, maximum fear $=4$ ); MPS presence (healthy athletes group $=0$, MPS athletes group $=1$ ).

$\mathrm{FABQ}=$ Fear Avoidance Beliefs Questionnaire; MPS = myofascial pain syndrome; TSK-11 = Tampa Scale of Kinesiophobia - 11 items.

$* P$ values $<0.05$ for a $95 \%$ confidence interval are shown.

$* * P$ values $<0.001$ for a $95 \%$ confidence interval are shown.

\section{Discussion}

To the authors' knowledge, this research study may be considered the first case-control study showing greater kinesiophobia levels, kinesiophobia total scores, and activity avoidance domain scores of the TSK-11 for athletes with gastrocnemius MPS compared with healthy athletes. Nevertheless, harm domain scores of TSK-11 did not show statistical differences between the groups. In addition, greater fear avoidance beliefs total scores and fear avoidance beliefs related to work and physical activity domains were present in athletes suffering from gastrocnemius MPS compared with healthy athletes. These findings were in accordance with previous studies evaluating kinesiophobia and fear avoidance beliefs in athletes suffering from other musculoskeletal conditions such as patellofemoral pain [6] and anterior cruciate ligament reconstruction [5]. In addition, prior studies have related MPS to kinesiophobia and fear avoidance beliefs, although in nonathletic populations and other myofascial pain conditions different from gastrocnemius such as temporomandibular disorders [48], fibromyalgia and chronic fatigue syndrome [49], low back pain, whiplash-associated disorders [50], migraine [51], and chronic mechanical neck pain [52].

Indeed, the activity avoidance domain of the TSK-11 showed statistically significant differences between both groups due to athletes with MPS presenting more beliefs that activities provoking soreness should be avoided compared with healthy athletes, whereas the harm domain of the TSK-11 did not show statistical differences between groups due to athletes presenting similar beliefs about soreness being a sign of bodily damage compared with healthy athletes [37-39]. A plausible explanation for these differences on kinesiophobia domains could be that fear of movement related to physical activity may play a key role in training and competition of athletes, whereas fear of movement related to harm could be a secondary issue for athletes who believe that soreness does not present a close relationship with bodily damage [5,6].

According to the first prediction model (Table 2), fear avoidance beliefs positively predicted kinesiophobia symptoms in athletes. These findings are in accordance with prior studies reporting that fear avoidance beliefs and kinesiophobia are inter-related and that their relationship plays a key role in athletes who suffer from pain conditions [5, 6]. Regarding the second prediction model (Table 4), the presence of gastrocnemius MPS, greater levels of fear of movement, and lower height predicted more fear avoidance beliefs in athletes. The presence of gastrocnemius MPS was shown as a powerful predictor of fear avoidance beliefs, which may be due to nociceptive inputs in athletes suffering 
from MPS increasing the neural synaptic excitability of the central nervous system nociceptive pathways [53], expanding referred pain patterns of active MTrPs in gait cycle propulsion muscles such as the gastrocnemius [7, 8], increasing fear avoidance beliefs and levels of fear of movement in athletes [5, 6]. Despite the lowest $R^{2}$ coefficient, lower height predicted higher fear avoidance beliefs in athletes. A prior study has reported a relationship between dynamic stability evaluated by functional reaching and multidirectional speed in athletes who present high movement demands or muscle recruitment [54]. Nevertheless, our findings relating height to fear avoidance beliefs have not been previously reported in the sport medicine literature, and further studies are needed in order to further explain this issue.

\section{Future Studies}

Future clinical trials should include kinesiophobia and fear avoidance beliefs as covariables to determine the clinical effectiveness of psychological or physical therapy treatments such as dry needling or ischemic compression in athletes suffering from gastrocnemius MPS [7, 8]. In addition, the influence of fear of movement and fear avoidance beliefs should be considered in future studies about clinical outcomes or return to sports in athletes suffering from chronic gastrocnemius MPS and Achilles tendinopathies [7, 55]. Finally, future studies should be carried out to determine foot orthoses' effectiveness in order to reduce kinesiophobia and fear avoidance beliefs in athletes with gastrocnemius MPS, as prior studies have shown a pain reduction in other musculoskeletal conditions such as low back pain [56], knee pain [57], forefoot pain [58], and rheumatoid arthritis [59].

\section{Limitations}

Some limitations should be acknowledged in the present study. First, the consecutive sampling method may be a limitation. Second, socioeconomic data and civil and working status were not collected. Finally, despite prior studies' recommendations mainly focused on MPS and active MTrPs according to the good reliability of diagnosis by a standardized manual palpation procedure [29], another possible limitation was that latent MTrPs' presence was not assessed in the present study and should be considered in the design of future studies, as latent MTrPs have been demonstrated to be a key focus of MPS sensitization [60].

\section{Conclusions}

Greater kinesiophobia levels, total scores, and activity avoidance domain scores (but not for the harm domain), as well as greater fear avoidance beliefs total scores and fear avoidance beliefs related to work and physical activity domains, were shown for athletes suffering from gastrocnemius MPS compared with healthy athletes. Indeed, higher kinesiophobia symptoms were predicted by greater fear avoidance beliefs in athletes. In addition, greater fear avoidance beliefs were predicted by the presence of gastrocnemius MPS, higher levels of kinesiophobia, and lower height in athletes.

\section{Authors' Contributions}

Concept: Marta San-Antolín, David Rodríguez-Sanz, Davinia Vicente-Campos, and César Calvo-Lobo; data curation: Marta San-Antolín; formal analysis: David Rodríguez-Sanz and César Calvo-Lobo; investigation: Marta SanAntolín, David Rodríguez-Sanz, Patricia Palomo-López, Carlos Romero-Morales, Maria Benito-De-Pedro, Daniel López-López, and César Calvo-Lobo; methodology: Marta San-Antolín, David Rodríguez-Sanz, Davinia VicenteCampos, Patricia Palomo-López, Carlos Romero-Morales, Maria Benito-De-Pedro, Daniel López-López, and César Calvo-Lobo; supervision: César Calvo-Lobo; writing—original draft: Marta San-Antolín, David Rodríguez-Sanz, Davinia Vicente-Campos, Patricia Palomo-López, Carlos Romero-Morales, Maria Benito-De-Pedro, Daniel López-López, and César Calvo-Lobo; writing - review \& editing: David Rodríguez-Sanz, Davinia Vicente-Campos, Patricia Palomo-López, Carlos Romero-Morales, Maria Benito-De-Pedro, Daniel López-López, and César Calvo-Lobo.

\section{References}

1. Simons DG, Travell JG, Simons LS, Travell JG. Travell \& Simons' Myofascial Pain and Dysfunction: The Trigger Point Manual. Williams \& Wilkins; 1999. [AQ8]

2. Fernández-de-las-Peñas C, Dommerholt J. International consensus on diagnostic criteria and clinical considerations of myofascial trigger points: A Delphi study. Pain Med 2018;19(1):142-50. 
3. Oosterhoff JHF, Bexkens R, Vranceanu A-M, Oh LS. Do injured adolescent athletes and their parents agree on the athletes' level of psychologic and physical functioning? Clin Orthop Relat Res 2018;476(4):767-75.

4. Goubert L, Crombez G, Van Damme S. The role of neuroticism, pain catastrophizing and pain-related fear in vigilance to pain: A structural equations approach. Pain 2004;107(3):234-41.

5. Hoch JM, Houston MN, Baez SE, Hoch MC. Fear-avoidance beliefs and health-related quality of life in post-ACL reconstruction and healthy athletes: A case-control study. J Sport Rehabil In press. [AQ9]

6. Priore LB, Azevedo FM, Pazzinatto MF, et al. Influence of kinesiophobia and pain catastrophism on objective function in women with patellofemoral pain. Phys Ther Sport 2019;35:116-21.

7. Benito-de-Pedro M, Becerro-de-Bengoa-Vallejo R, Elena Losa-Iglesias M, et al. Effectiveness of deep dry needling vs ischemic compression in the latent myofascial trigger points of the shortened triceps surae from triathletes on ankle dorsiflexion, dynamic, and static plantar pressure distribution: A clinical trial. Pain Med 2019;XXX(X):XXX$\mathrm{XX}$

8. E and ischemic compression in the triceps surae latent myofascial trigger points of triathletes on pressure pain threshold and thermography: A single blinded randomized clinical trial. J Clin Med 2019;8(10). [AQ10]

9. Dommerholt J. Dry needling — peripheral and central considerations. J Man Manip Ther 2011;19(4):223-7.

10. Ge H-Y, Fernández-de-Las-Peñas C, Yue S-W. Myofascial trigger points: Spontaneous electrical activity and its consequences for pain induction and propagation. Chin Med 2011;6(1):13.

11. Bron C, Dommerholt JD. Etiology of myofascial trigger points. Curr Pain Headache Rep 2012;16(5):439-44.

12. Grieve R, Barnett S, Coghill N, Cramp F. The prevalence of latent myofascial trigger points and diagnostic criteria of the triceps surae and upper trapezius: A cross sectional study. Physiotherapy 2013;99(4):278-84.

13. Grieve R, Barnett S, Coghill N, Cramp F. Myofascial trigger point therapy for triceps surae dysfunction: A case series. Man Ther 2013;18(6):519-25.

14. Ahmad CS, Dick RW, Snell E, et al. Major and minor league baseball hamstring injuries: Epidemiologic findings from the Major League Baseball Injury Surveillance System. Am J Sports Med 2014;42(6):1464-70.

15. Bertola IP, Sartori RP, Corrêa DG, Zotz TGG, Gomes A. Profile of injures prevalence in athletes who participated in SESC Triathlon Caiobá-2011. Acta Ortop Bras 2014;22(4):191-6.

16. Martín-Pintado-Zugasti A, López-López A, González Gutiérrez JL, et al. The role of psychological factors in the perception of postneedling soreness and the influence of postneedling intervention. 2017;9:348-55. [AQ11]

17. Fernández-Carnero J, Gilarranz-de-Frutos L, León-Hernández JV, et al. Effectiveness of different deep dry needling dosages in the treatment of patients with cervical myofascial pain: A pilot RCT. Am J Phys Med Rehabil 2017;96(10):726-33.

18. Martín-Pintado-Zugasti A, Fernández-Carnero J, León-Hernández JV, et al. Postneedling soreness and tenderness after different dosages of dry needling of an active myofascial trigger point in patients with neck pain: A randomized controlled trial. PM R 2018;10(12):1311-20.

19. Kennedy JG, Knowles B, Dolan M, Bohne W. Foot and ankle injuries in the adolescent runner. Curr Opin Pediatr 2005;17(1):34-42.

20. Vandenbroucke JP, von Elm E, Altman DG, et al. Strengthening the Reporting of Observational Studies in Epidemiology (STROBE): Explanation and elaboration. Int J Surg 2014;12(12):1500-24.

21. San-Antolín M, Rodríguez-Sanz D, Becerro-de-Bengoa-Vallejo R, et al. Central sensitization and catastrophism symptoms are associated with chronic myofascial pain in the gastrocnemius of athletes. Pain Med 2019; XXX(X):XXX-XX

22. World Medical Assoc.... Declaration of Helsinki. Ethical principles for medical research involving human subjects. J Am Coll Dent 2014;81(3):14-8. 
23. Faul F, Erdfelder E, Lang A-G, Buchner A. G*Power 3: A flexible statistical power analysis program for the social, behavioral, and biomedical sciences. Behav Res Methods 2007;39(2):175-91.

24. Gómez-Pérez L, López-Martínez AE, Ruiz-Párraga GT. Psychometric properties of the Spanish version of the Tampa Scale for Kinesiophobia (TSK). J Pain 2011;12(4):425-35. [AQ12]

25. Woby SR, Roach NK, Urmston M, Watson PJ. Psychometric properties of the TSK-11: A shortened version of the Tampa Scale for Kinesiophobia. Pain 2005;117(1):137-44.

26. Hagstromer M, Oja P, Sjostrom M. The International Physical Activity Questionnaire (IPAQ): A study of concurrent and construct validity. Public Health Nutr 2006;9(6):755-62.

27. Romero-Morales C, Almazán-Polo J, Rodríguez-Sanz D, et al. Rehabilitative ultrasound imaging features of the abdominal wall muscles in elite and amateur basketball players. Appl Sci 2018;8(5):809.

28. Calvo-Lobo C, Almazán-Polo J, Becerro-de-Bengoa-Vallejo R, et al. Ultrasonography comparison of diaphragm thickness and excursion between athletes with and without lumbopelvic pain. Phys Ther Sport 2019;37:128-37.

29. Myburgh C, Lauridsen HH, Larsen AH, Hartvigsen J. Standardized manual palpation of myofascial trigger points in relation to neck/shoulder pain; the influence of clinical experience on inter-examiner reproducibility. Man Ther 2011;16(2):136-40.

30. Pecos-Martín D, Montañez-Aguilera FJ, Gallego-Izquierdo T, et al. Effectiveness of dry needling on the lower trapezius in patients with mechanical neck pain: A randomized controlled trial. Arch Phys Med Rehabil 2015;96(5):775-81.

31. Lobo CC, Morales CR, Sanz DR, Corbalán IS, Marín AG, López DL. Ultrasonography comparison of peroneus muscle cross-sectional area in subjects with or without lateral ankle sprains. J Manipulative Physiol Ther 2016;39(9):635-44.

32. Garrow JS. Quetelet index as indicator of obesity. Lancet 1986;1(8491):1219.

33. Boonstra AM, Schiphorst Preuper HR, Reneman MF, Posthumus JB, Stewart RE. Reliability and validity of the visual analogue scale for disability in patients with chronic musculoskeletal pain. Int J Rehabil Res 2008;31(2).

34. Craig CL, Marshall AL, Sjöström M, et al. International Physical Activity Questionnaire: 12-country reliability and validity. Med Sci Sports Exerc 2003;35(8):1381-95.

35. García Campayo J, Rodero B, Alda M, Sobradiel N, Montero J, Moreno S. Validation of the Spanish version of the Pain Catastrophizing Scale in fibromyalgia. Med Clin (Barc) 2008;131(13):487-92.

36. Sullivan MJL, Bishop SR, Pivik J. The Pain Catastrophizing Scale: Development and validation. Psychol Assess 1995;7(4):524-32.

37. Swinkels-Meewisse IEJ, Roelofs J, Schouten EGW, Verbeek ALM, Oostendorp RAB, Vlaeyen J. Fear of movement/(re)injury predicting chronic disabling low back pain: A prospective inception cohort study. Spine (Phila Pa 1976) 2006;31(6):658-64.

38. Ferrer-Peña R, Moreno-López M, Calvo-Lobo C, López-de-Uralde-Villanueva I, Fernández-Carnero J. Relationship of dynamic balance impairment with pain-related and psychosocial measures in primary care patients with chronic greater trochanteric pain syndrome. Pain Med 2019;20(4):810-7.

39. Costa Lda CM, Maher CG, McAuley JH, Hancock MJ, Smeets R. Self-efficacy is more important than fear of movement in mediating the relationship between pain and disability in chronic low back pain. Eur J Pain 2011;15(2):213-9.

40. Chmielewski TL, Zeppieri G, Lentz TA, et al. Longitudinal changes in psychosocial factors and their association with knee pain and function after anterior cruciate ligament reconstruction. Phys Ther 2011;91(9):1355-66.

41. George SZ, Valencia C, Beneciuk JM. A psychometric investigation of fear-avoidance model measures in patients with chronic low back pain. J Orthop Sports Phys Ther 2010;40(4):197-205. 
42. Hapidou EG, O'Brien MA, Pierrynowski MR, de las Heras E, Patel M, Patla T. Fear and avoidance of movement in people with chronic pain: Psychometric properties of the 11-item Tampa Scale for Kinesiophobia (TSK-11). Physiother Can 2012;64(3):235-41.

43. Waddell G, Newton M, Henderson I, Somerville D, Main CJ. A Fear-Avoidance Beliefs Questionnaire (FABQ) and the role of fear-avoidance beliefs in chronic low back pain and disability. Pain 1993;52(2):157-68.

44. Mintken PE, Cleland JA, Whitman JM, George SZ. Psychometric properties of the fear-avoidance beliefs questionnaire and Tampa Scale of Kinesiophobia in patients with shoulder pain. Arch Phys Med Rehabil 2010;91(7):1128-36.

45. Kovacs FM, Muriel A, Medina JM, Abraira V, Castillo Sánchez MD, Jaúregui JO. Psychometric characteristics of the Spanish version of the FAB questionnaire. Spine (Phila Pa 1976) 2006;31(1):104-10.

46. Kelley K, Preacher KJ. On effect size. Psychol Methods 2012;17(2):137-52.

47. Austin PC, Steyerberg EW. The number of subjects per variable required in linear regression analyses. J Clin Epidemiol 2015;68(6):627-36.

48. Gil-Martínez A, Grande-Alonso M, López-de-Uralde-Villanueva I, López-López A, Fernández-Carnero J, La Touche R. Chronic temporomandibular disorders: Disability, pain intensity and fear of movement. J Headache Pain 2016;17(1):103.

49. Malfliet A, Van Oosterwijck J, Meeus M, et al. Kinesiophobia and maladaptive coping strategies prevent improvements in pain catastrophizing following pain neuroscience education in fibromyalgia/chronic fatigue syndrome: An explorative study. Physiother Theory Pract 2017;33(8):653-60.

50. Reis F, Guimarães F, Nogueira LC, Meziat-Filho N, Sanchez TA, Wideman T. Association between pain drawing and psychological factors in musculoskeletal chronic pain: A systematic review. Physiother Theory Pract 2019;35(6):533-42.

51. Benatto MT, Bevilaqua-Grossi D, Carvalho GF, et al. Kinesiophobia is associated with migraine. Pain Med 2019;20(4):846-51.

52. Saavedra-Hernández M, Castro-Sánchez AM, Cuesta-Vargas AI, Cleland JA, Fernández-de-las-Peñas C, ArroyoMorales M. The contribution of previous episodes of pain, pain intensity, physical impairment, and pain-related fear to disability in patients with chronic mechanical neck pain. Am J Phys Med Rehabil 2012;91(12):1070-6.

53. Graven-Nielsen T, Arendt-Nielsen L. Assessment of mechanisms in localized and widespread musculoskeletal pain. Nat Rev Rheumatol 2010;6(10):599-606.

54. Lockie RG, Schultz AB, Callaghan SJ, Jeffriess MD. The relationship between dynamic stability and multidirectional speed. J Strength Cond Res 2016;30(11):3033-43.

55. Usuelli F, D’Ambrosi R, Manzi L, Indino C, Villafañe J, Berjano P. Clinical outcomes and return to sports in patients with chronic Achilles tendon rupture after minimally invasive reconstruction with semitendinosus tendon graft transfer. Joints 2017;05(04):212-6.

56. Castro-Méndez A, Munuera PV, Albornoz-Cabello M. The short-term effect of custom-made foot orthoses in subjects with excessive foot pronation and lower back pain: A randomized, double-blinded, clinical trial. Prosthet Orthot Int 2013;37(5):384-90.

57. Munuera PV, Mazoteras-Pardo R. Benefits of custom-made foot orthoses in treating patellofemoral pain. Prosthet Orthot Int 2011;35(4):342-9.

58. Arias-Martín I, Reina-Bueno M, Munuera-Martínez PV. Effectiveness of custom-made foot orthoses for treating forefoot pain: A systematic review. Int Orthop 2018;42(8):1865-75.

59. Reina-Bueno M, Vázquez-Bautista MDC, Pérez-García S, Rosende-Bautista C, Sáez-Díaz A, Munuera-Martínez PV. Effectiveness of custom-made foot orthoses in patients with rheumatoid arthritis: A randomized controlled trial.

Clin Rehabil 2019;33(4):661-9. 
60. Fernández-Carnero J, Fernández-de-las-Peñas C, de la Llave-Rincón AI, Ge H-Y, Arendt-Nielsen L. Bilateral myofascial trigger points in the forearm muscles in patients with chronic unilateral lateral epicondylalgia. Clin $\mathrm{J}$ Pain 2008;24(9):802-7.

\section{AUTHOR QUERIES}

Query: AQ1: Please confirm whether the article title is OK as set. Response: Accept

Query: AQ2: Please check all author names and affiliations. Please check that author surnames have been identified by a pink background. This is to ensure that forenames and surnames have been correctly tagged for online indexing.

Response: Please, change affiliation of César Calvo-Lobo, PhD, MSc, PT by "†Facultad de Enfermería, Fisioterapia y Podología, Universidad Complutense de Madrid, Madrid, Spain"

Query: AQ3: Please provide a full mailing address and telephone and fax numbers for the corresponding author. Response: Accept

Query: AQ4: Please check the usage of both instances of the word "showed" here.

Response: Accept

Query: AQ5: This text has been edited heavily for English language and grammar. Please check thoroughly to ensure that the intended meaning has been retained throughout.

Response: Accept

Query: AQ6: If your manuscript has figures or text from other sources, please ensure you have permission from the copyright holder. For any questions about permissions contact jnls.author.support@oup.com.

Response: Accept

Query: AQ7: Please check the edits to this sentence.

Response: Accept

Query: AQ8: Please provide the publisher location for ref. 1.

Response: Baltimore, MD,

Query: AQ9: Please provide updated publication information (if available) for all "in press" references.

Response: Please, update as "Hoch JM, Houston MN, Baez SE, Hoch MC. Fear-Avoidance Beliefs andHealth-Related Quality of Life in Post-ACL Reconstruction and Healthy Athletes: ACase-Control Study. J Sport Rehabil. 2019 Oct 18:1-5. doi: 10.1123/jsr.2018-0491."

Query: AQ10: Please provide a full page range for refs. 8, 10, 27, 32, 33, 48.

Response: Please, update (page 1632) as "J. Clin. Med. 2019, 8(10), 1632; https://doi.org/10.3390/jcm8101632"

Query: AQ11: Please provide the journal abbreviation for ref. 16.

Response: PM R

Query: AQ12: The details given in references 24 and 40 were the same. Hence, reference 40 has been deleted, and references and citations have been renumbered accordingly. Please check.

Response: Accept

Query: AQ13: Please provide one or more in-text citation(s) for Figure 2.

Response: You can add references "[24,25]"

Query: AQ14: These figures are currently intended to appear online in colour and black and white in print. Please reword the legend/text to avoid using reference to colour. Alternatively, please let us know if you wish to pay for print colour reproduction or to have both versions in black and white. The standard charge for colour reproduction in print is $£ 350 / e 525 / \$ 600$ per figure. Please check the black and white versions at the end of the paper and contact us if you have any concerns. If there are no color figures in your article, please ignore this query 
Response: Thanks. You can use online in colour and black and white in print. We do not want to pay for colour reproduction.

\section{COMMENTS}

C1 Author: Remove "]";

C2 Author: ;

C3 Author: ; 